\title{
Botulinum toxin: A potential alternative to current treatment of neurogenic and idiopathic urinary incontinence due to detrusor overactivity
}

\author{
N. Casanova ${ }^{a, 1}$, E. McGuire ${ }^{\text {b, } 1}$, D.E. Fenner ${ }^{a, *}$ \\ a University of Michigan, Department of Obstetrics and Gynecology, Ann Arbor, MI, USA \\ b University of Michigan, Department of Urology, Ann Arbor, MI, USA
}

Accepted 30 August 2006

\section{KEYWORDS \\ Botulinum toxin; Neurogenic bladder; Detrusor overactivity}

\begin{abstract}
Objective: To analyze and report the current data on the treatment of both neurogenic and idiopathic detrusor overactivity with Botulinum toxin. Methods: Literature review using Pub-Med and Medline from 1990 until June 30, 2006. Results: Case series of patients with neurogenic detrusor dysfunction (NDD) and idiopathic detrusor overactivity (IDO) range from 15 to 200 patients with follow up from 12 to 36 weeks post-treatment. Significant improvements in cystometric bladder capacity, reflex volume at first urge to void, and bladder compliance are seen in nearly all patients. Approximately 50\% of NDD patients achieved urinary continence and almost all had improvement in bladder control up to 36 weeks following treatment. Patients with IDO with urgency alone or with incontinence also had urodynamic as well as symptom improvement. Approximately $75 \%$ of patients with IDO and incontinence are dry at 12 weeks post-treatment. Urgency disappears on average in two thirds of patients. Quality of life scores also shows significant improvement for all groups. Conclusion: Botulinum toxin-A has emerged as a promising option for the treatment of neurogenic and refractory idiopathic detrusor overactivity. Studies to date have shown that not only is this treatment effective at decreasing urinary symptoms and incontinence, as well as improving potentially dangerous urodynamic measures, but it is also minimally invasive, reversible and safe. Questions over proper dosing and dilution, number of injection sites, and re-injection rates remain to be answered.

(c) 2006 International Federation of Gynecology and Obstetrics. Published by Elsevier Ireland Ltd. All rights reserved.
\end{abstract}

\section{Background/Introduction}

* Corresponding author. Tel.: +1 7346479727.

E-mail address: deef@umich.edu (D.E. Fenner).

1 Tel.: +1 7346479727.
Botulinum toxin, first isolated in 1897 , is synthesized from the gram positive anaerobic bacteria, Clostridium botulinum, and is the most potent naturally

0020-7292/\$ - see front matter ๑ 2006 International Federation of Gynecology and Obstetrics. Published by Elsevier Ireland Ltd. All rights reserved.

doi:10.1016/j.ijgo.2006.08.014 
occurring neurotoxin known to man. This bacterium is usually found in soil and can produce one of 7 distinct toxins labeled A-G [1]. Currently the toxin subtypes A and $B$ have been clinically implicated in the treatment of several neuromuscular conditions and have been FDA approved in the treatment of blepharospasm, strabismus, and wrinkles [2]. Currently use of Botulinum toxin for neurogenic detrusor dysfunction and idiopathic overactive bladder is off-label use of this medication in the United States.

The toxin complex itself ranges from 300 to $900 \mathrm{kDa}$, which includes a $150 \mathrm{kD}$ exotoxin, complete with both a heavy and a light chain. This complex exerts its action by heavy chain binding to a neuron, leaving the light chain to cleave a specific site on the target protein complex. Specifically, the light chain of Botulinum toxin-A (BTX-A) cleaves the synaptosomal associated SNAP-25 protein within the SNARE complex, which is involved in the exocytosis of acetylcholine vesicles in peripheral motor neurons. Upon execution of this cleavage, the motor neuron is paralyzed, thereby inhibiting contraction and causing muscle relaxation [3]. Overall, this chemodenervation is fully reversible as the formation of functional neuronal sprouts reconnects nerve endings in anywhere from 3 to 9 months on average [1]. In addition to its reversibility, the response is both dose and site specific, giving a safe and effective result.

Given the effect of Botulinum toxin on neuromuscular activity and both its reversibility and safety, the interest in its therapeutic applications has grown over the years to include indications such as juvenile cerebral palsy, focal spasticity, cervical dystonia and most importantly for this review, detrusor overactivity. In recent years preliminary data suggests that this may be effective for both neurogenic (NDO) and idiopathic detrusor overactivity (IDO) in decreasing bladder pressures, frequency and incontinence episodes as well as increasing bladder capacity. This discovery is quite important in that there is certainly a need for alternative treatments for bladder dysfunction. The side effects and effectiveness profiles of current conservative measures, such anticholinergic medications, plus the complications associated with more definitive measures such as bladder augmentation, encourage physicians and scientists to seek new alternative therapies.

The procedure itself is usually performed under a general or local anesthetic if bladder sensation is intact. A $22 \mathrm{~F}$ rigid cystoscope with a flexible 23 gauge injection needle is then inserted into the bladder and anywhere from $100 \mathrm{U}$ to $300 \mathrm{U}$ of Botulinum toxin diluted in saline is injected in 15-30 different sites in the bladder. The trigone is usually spared given its extensive innervation and the risk of urinary retention. The injections usually begin to take effect within 3-
7 days and can last to an average of 6 months [3]. (Refer to http://www.med.umich.edu/lrc/pva/ for a video of the procedure.)

The goal of this review is to analyze and report the current data on the treatment of both neurogenic and idiopathic detrusor overactivity with Botulinum toxin and to look at what the future of this potentially revolutionary treatment holds.

\section{Neurogenic detrusor overactivity}

Involuntary detrusor contractions, as a result of neurologic pathway interruption, are the hallmark of the neurogenic bladder dysfunction [4]. As a result of these uninhibited contractions, pressures in the bladder can rise to dangerous levels, predisposing patients to infection, causing incontinence and, if severe, causing vesicoureteral reflux and renal damage. Currently, anticholinergic medications, clean intermittent catheterization and indwelling catheters are used as conservative treatment measures, but side effects of the medications, complications related to indwelling catheters and refractory symptoms are often indications for more definitive measures. When conservative measures fail, more invasive procedures such as sacral root rhizotomy, pudendal nerve stimulation, and bladder augmentation are usually the next line of defense. However, these measures come with quite a few complications of their own, making them a less favorable option and leaving both physicians and patients looking for alternative treatment methods.

Over the last few years, the use of Botulinum toxin-A has been one of the front running contenders in the search for a more suitable treatment. In a early study reported in 2000 by Schurch et al. [5] (Table 1), 21 patients were evaluated at 6,16 , and 36 weeks after either 200 or $300 \mathrm{U}$ injections of BTX-A. At the 6 week follow up, 17 of the 19 patients evaluated were fully continent and at 36 weeks, continence persisted in 7 of the 11 remaining patients seen in follow up, without significant complications. Interestingly, the 2 patients who failed treatment and continued to have incontinence at 6 weeks, received only $200 \mathrm{U}$ of the toxin vs. the higher $300 \mathrm{U}$ dose. In addition to symptomatic improvement, bladder parameters on urodynamic testing improved in all patients at all included time points. Mean cystometric bladder capacity (MCBC) increased from $296.3 \mathrm{cc}$ to $480.5 \mathrm{cc}$ and $457.5 \mathrm{cc}$ at 6 and 36 weeks respectively as the mean reflex volume at first urge to void (RV) also increased from $215.8 \mathrm{cc}$ at baseline, to $415.7 \mathrm{cc}$ and $319.6 \mathrm{cc}$ at 6 and 36 weeks. This study also demonstrated increases in the mean bladder compliance $(M B C)$ and decreases in the mean maximum detrusor pressure during uninhibited contractions (MVP), which also persisted at 36 weeks. 


\begin{tabular}{|c|c|c|c|c|c|c|c|c|}
\hline \multirow[t]{2}{*}{ Study } & \multirow[t]{2}{*}{$N=$} & \multirow[t]{2}{*}{$\mathrm{F} / \mathrm{U}$} & \multirow[t]{2}{*}{ Dose/inj \# } & \multicolumn{2}{|c|}{ MCBC (cC) } & \multicolumn{2}{|c|}{$\begin{array}{l}\text { Reflex } \\
\text { volume (cc) } \\
\end{array}$} & \multirow[t]{2}{*}{ Continence/outcomes } \\
\hline & & & & Pre & Post & Pre & Post & \\
\hline Schurch et al. [5] & 21 & 36 weeks & $200-300 / 20-30$ & 296 & 457.5 & 215.8 & 319.6 & $\begin{array}{l}-17 / 19 \text { continent at } 6 \text { weeks } \\
-7 / 11 \text { continent at } 36 \text { weeks } \\
-7 \mathrm{~d} / \mathrm{c} \text { meds, } 10 \text { dec by } 20-50 \%\end{array}$ \\
\hline Bagi and Beiring-Sorenson [6] & 15 & 6 weeks & $300 / 30$ & 350 & 457 & & & $\begin{array}{l}13 / 15 \text { cont, } 2 \text { reduced leak, } \\
\text { mean } 7 \text { mo cont }\end{array}$ \\
\hline Reitz et al. [3] & 200 & 36 weeks & $300 \mathrm{U} / 30$ & 272 & 352 & 236 & 291 & $\begin{array}{l}-132 / 180 \text { continent } 12 \text { weeks } \\
-72 / 99 \text { continent at } 36 \text { weeks } \\
-45 \mathrm{~d} / \mathrm{c} \text { meds, and } 118 \text { reduced } \\
\text { medication use }\end{array}$ \\
\hline Klaphajone et al. [7] & 10 & 36 weeks & $300 \mathrm{U} / 15-30$ & 175 & 230 & 54 & $\sim 70$ & $\begin{array}{l}-7 / 10 \text { continent at } 6 \text { weeks } \\
-5 / 10 \text { continent at } 36 \text { weeks }\end{array}$ \\
\hline Schurch et al. [8] & 59 & 24 weeks & $\begin{array}{l}a-300 / 30 \\
b-200 / 30\end{array}$ & $\begin{array}{l}a-293 \\
b-260\end{array}$ & $\begin{array}{l}a-398 \\
b-441\end{array}$ & $\begin{array}{l}a-255 \\
b-169\end{array}$ & $\begin{array}{l}a-305 \\
b-327\end{array}$ & $\begin{array}{l}\text { I-QOL trial: all improved } 50 \% \\
\text { reduction in incont. rates }\end{array}$ \\
\hline
\end{tabular}

Another study involving 15 patients [6] confirmed the above improvements, reporting continence in 13 of their 15 study participants at 6 weeks post-injection. Each patient's urodynamic parameters showed significant changes, including decreases in their maximum detrusor pressures and significant increases in their maximum volume at safe detrusor pressures of $<40 \mathrm{cmH}_{2} \mathrm{O}$ (from a mean of $185 \mathrm{cc}$ to $457 \mathrm{cc}$ ). These results persisted at the 24 week follow up time.

The largest study was reported in 2004, and is regarded as the current "gold standard". Over 200 patients from different institutions across Europe similarly demonstrated significant improvements in both symptoms and urodynamic parameters in patients with neurogenic bladder dysfunction treated with BTX-A [3]. This study followed patients at 12 and 36 weeks after injection with $300 \mathrm{U}$ of the toxin at 30 different injection sites. Of the 180 patients examined at 12 weeks, 132 of them reported complete continence, while the remaining 48 patients reported significant improvement in their symptoms $(73 \%$ continence rate). At 36 weeks, 72/99 remaining patients reported continence. Additionally, at 12 weeks, 45 patients were able to completely discontinue their anticholinergic medications while 118 were able to significantly reduce their medication use. Moreover, no significant complications were noted as a result of the injections. From a urodynamic standpoint, results were likewise quite impressive. The mean MCBC increased significantly from a baseline of $272 \mathrm{cc}$ to $420 \mathrm{cc}$ and $352 \mathrm{cc}$ at 12 and 36 weeks respectively. The RV increased from $236 \mathrm{cc}$ to $387 \mathrm{cc}$ and $291 \mathrm{cc}$ at 12 and 36 weeks. And, much like the Schurch study in 2000 , this study also demonstrated significant beneficial changes in MVP and MBC during follow up evaluations, overall supporting the theory that BTX-A toxin may be quite beneficial in the treatment of neurogenic detrusor dysfunction.
Although many of the aforementioned studies did show an overall increase in bladder compliance, none of those papers specifically examined patients with abnormally low compliance bladders. This special population was studied by Klaphajone et al. [7] in 2005. This study evaluated the effects of 300 U BTX-A injections in 10 patients with spinal cord injuries and resultant abnormally low compliance bladders. During follow up evaluations, bladder compliance was shown to increase significantly from $6.5 \mathrm{cc} / \mathrm{cmH}_{2} \mathrm{O}$ to approximately $14.5 \mathrm{ml} / \mathrm{cmH}_{2} \mathrm{O}$ at 6 and 16 weeks with a return closer to baseline at 36 weeks. Results were similar for MCBC and RV as initial evaluations at 6 weeks showed a mean MCBC to increase from $175 \mathrm{cc}$ to $331 \mathrm{cc}$ and RV from $54 \mathrm{cc}$ to $86 \mathrm{cc}$. Again, these results were maintained at 16 weeks but returned close to baseline at 36 weeks.

While the results of these case series reported above are quite encouraging for the use of BTX-A in the treatment of neurogenic bladder overactivity, the lack of controls and quality of life outcomes are major limitations of these trials. One study, reported in 2005 [8], however attempts to relieve these limitations by creating a randomized controlled trial to evaluate treatment. In this study a total of 59 matched patients were randomized into three groups receiving $200 \mathrm{U}$ BTX-A, $300 \mathrm{U}$ BTX-A or placebo injections and were evaluated at various time points for a total of 24 weeks. Compared with placebo, significant clinical and urodynamic improvements were demonstrated in the 2 BTX-A groups. Among the measured outcomes were incontinence episodes and quality of life profiles using the validated I-QOL questionnaire. Between the 2 BTX-A groups, there was an overall continence rate of $50 \%$ with marked improvement in I-QOL scores compared with placebo at all evaluated time points (Fig. 1). MCBC and RV also improved significantly in the BTX-A treatment groups 


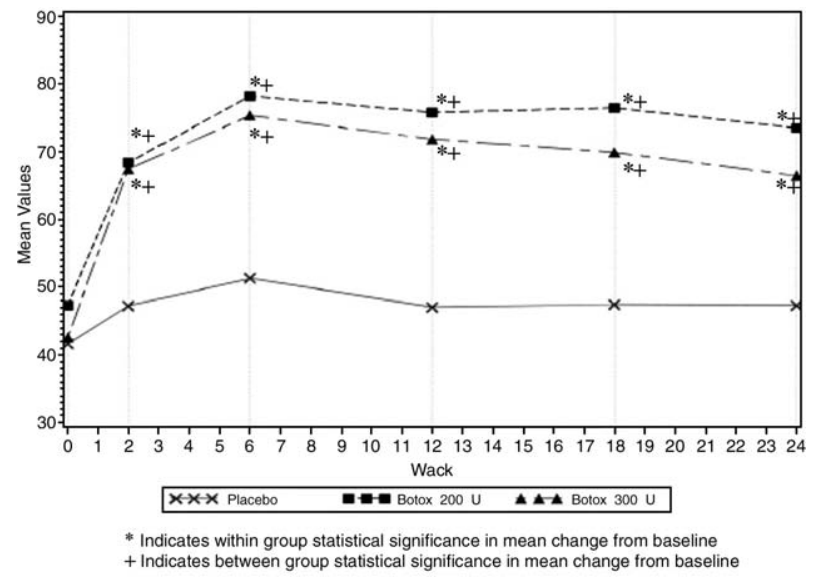

Figure 1 I-QOL scores in placebo vs. BTX-A treatment groups. Data from this figure are taken from Ref. [9].

citing increases in mean MCBC from $293 \mathrm{cc}$ at baseline to $398 \mathrm{cc}$ and RV from $255 \mathrm{cc}$ to $305 \mathrm{cc}$. Much safer maximum detrusor pressures were also achieved with a decrease in MVP from an average of $92 \mathrm{cmH}_{2} \mathrm{O}$ at baseline, to $55 \mathrm{cmH}_{2} \mathrm{O}$ at 24 weeks. There was no statistical difference in efficacy between the two BTX-A groups in outcome measures. Additionally this treatment was deemed quite safe as no adverse events were considered to be related to the study medication. While this study clearly supports previous investigations, it is one of the only randomized controlled trials examining this hypothesis, thus lending strength to the overall data.

\section{Idiopathic detrusor overactivity: overactive bladder (OAB)}

Overactive bladder syndrome $(O A B)$ is defined as urgency with or without urge incontinence that is usually associated with frequency and nocturia. It is usually idiopathic in origin and is a condition that affects over $17 \%$ of the American and European populations [9]. Much like neurogenic bladder dysfunction, this condition is characterized by frequent uninhibited contractions of the bladder but due to the intact ability to void with urge; this does not usually result in dangerous pressure elevations within the bladder [4]. The symptoms, however, create quite an impact on patient quality of life and over the past decade more and more patients have come forward, looking for appropriate management. Current management focuses on pharmacologic measures such as anticholinergic medications vs. invasive surgical procedures to decrease symptoms. Given the limitations of medical treatment such as a high side effect profile and incomplete effectiveness, investigators are searching for alternative measures. Since preliminary data suggested that Botulinum toxin-A may be effective in treating uninhibited neurogenic bladder contractions, investigators postulated that the toxin may be effective in treating idiopathic bladder dysfunction as well. Studies now show that in-fact, not only do the toxin injections decrease symptoms, even in cases refractory to medications, but they also improve urodynamic parameters. In a study performed by Werner et al. in 2005 [10], 26 women with urge incontinence and urodynamic evidence of detrusor overactivity were followed for 36 weeks after injection of $100 \mathrm{U}$ of BTX-A toxin. After one week, patients were asked to decrease their use of anticholinergic medications and subsequently, voiding diaries and urodynamics were evaluated at 4, 12, and 36 weeks after injection. Overall, improvements were demonstrated in urodynamic parameters, urge symptoms, and quality of life. At 12 and 36 weeks respectively, the mean MCBC increased from a baseline of $216 \mathrm{cc}$ to $351.9 \mathrm{cc}$ and $340.4 \mathrm{cc}$ (Table 2) and the volume at first desire to void increased from $116.8 \mathrm{cc}$ to $192.2 \mathrm{cc}$ and $171.4 \mathrm{cc}$ at 12 and 36 weeks. At 4 weeks, 18/26 (69\%) of patients reported continence and at 12 weeks, 16/20 were continent. However, at 36 weeks, only $1 / 5$ remained continent. (All other patients were lost to follow up.) Urge symptoms reportedly improved as well, as the average daytime frequency decreased from 11.7 episodes per day to 7.2 and 6.6 at 4 and

Table 2 Outcomes of Botulinum toxin-A injections on idiopathic detrusor overactivity

\begin{tabular}{|c|c|c|c|c|c|c|c|c|}
\hline \multirow[t]{2}{*}{ Study } & \multirow[t]{2}{*}{$N=$} & \multirow[t]{2}{*}{$T: f / u$} & \multirow[t]{2}{*}{ Dose/inj \# } & \multicolumn{2}{|c|}{$\mathrm{MCBC}(\mathrm{cC})$} & \multirow{2}{*}{\multicolumn{2}{|c|}{$\begin{array}{l}\text { Volume @ } \\
\text { first urge } \\
\text { to void (cc) }\end{array}$}} & \multirow[t]{2}{*}{ Continence/outcomes } \\
\hline & & & & Pre & Post & & & \\
\hline Werner et al. [10] & 26 & 36 weeks & $100 \mathrm{U} / 30$ & 216 & 340.4 & 116.8 & 171.4 & $\begin{array}{l}16 / 20 \text { continent at } 12 \text { weeks, small } \\
\text { sample size at } 36 \text { weeks }\end{array}$ \\
\hline Kuo [11] & 20 & 24 weeks & $200 \mathrm{U} / 40$ & 325.1 & 371.2 & 139.8 & 208.2 & $7 / 20$ continent with improvement in 8 \\
\hline $\begin{array}{l}\text { Schulte-Baukloh } \\
\text { et al. [12] }\end{array}$ & 7 & 24 weeks & $300 \mathrm{U} / 40$ & 224.4 & 307.6 & 149 & 240 & 5/7: improved sx control \\
\hline Schmid et al. [9] & 100 & 12 weeks & $100 \mathrm{U} / 30$ & 246 & 381 & 126 & 212 & $\begin{array}{l}88 \% \text { improvement in } \mathrm{KHQ} \text { at } 36 \text { weeks: } \\
\text { still improvements but diminished vs. } \\
16 \text { weeks }\end{array}$ \\
\hline
\end{tabular}


12 weeks and average nocturia decreased from 2.6 episodes per night to 1.2 at both 4 and 12 weeks. Additionally, quality of life, as evaluated by the King's Health Questionnaire (KHQ), significantly improved at both 4 and 12 weeks, but statistical analyses were not done at 36 weeks due to the small sample size. No serious complications or instances of urinary retention were reported, however 2 patients did develop elevated PVRs at 4 weeks and were instructed to selfcatheterize for one week, or until the PVR was less than 100 . Overall, this study concluded that while BTX-A seems to be both safe and effective in the treatment of $O A B$, many questions, such as dosage, number of injections and injection rate, remain unanswered.

By looking at other studies, the question of dose safety and efficacy may be informally addressed. A study reported in 2005 by Kuo [11] evaluated 20 women with OAB after injection of $200 \mathrm{U}$ of BTX-A toxin vs. the $100 \mathrm{U}$ used in the previous study. At the 12 and 24 week follow up times, mean MCBC significantly increased from $224.4 \mathrm{cc}$ to $314.9 \mathrm{cC}$ and $307.6 \mathrm{cc}$ respectively and the volume at first sensation to void increased from a baseline of 139.8 cc to 212 cc and 208.2 cc at 12 and 24 weeks. Detrusor pressures also improved, showing pressure decreases from $29.8 \mathrm{cmH}_{2} \mathrm{O}$ to 21.5 and 24.1 at 12 and 24 weeks respectively. Subjective efficacy was also noted as continence at 12 weeks was achieved in 9 of 20 patients with 8 reporting improvement and at 24 weeks 7 remained continent and 8 continued to report improvement. Although efficacy was certainly demonstrated in this study, side effects such as urinary retention and elevated PVRs were seen as well. At 2 weeks, 6 patients demonstrated transient urinary retention, 10 had elevated PVRs and 15 patients $(75 \%)$ developed difficulty urinating and a sensation of residual urine after voiding. Although the majority of these adverse effects receded after 2 weeks, 6 patients continued to have PVRs of at least $1 / 3$ their total bladder capacity at 24 weeks. Taken as a whole, this study does support the use of BTX-A in the treatment of detrusor overactivity, but suggests that in a population of patients with intact bladder sensation, an elevated dose may cause transient urinary retention and elevated PVRs leading researchers to believe that a lower dose may be more appropriate.

In addition to its use in the treatment of detrusor overactivity, BTX-A has also shown to be effective at improving outcomes and satisfaction in patients with overactive bladder symptoms without detrusor overactivity. In a study performed by Schulte-Baukloh et al. [12], 7 patients with overactive bladder symptoms refractory to conservative management were injected with $300 \mathrm{U}$ of BTX-A and followed, evaluating urodynamic parameters and subjective measures of incontinence at 4, 12, and 24 weeks. Urodynamic evaluation showed an increase in the volume at first desire to void from $149 \mathrm{cc}$ to $230 \mathrm{cc}$ at 12 weeks and a significant increase to $240 \mathrm{cc}$ at 24 weeks. The MCBC also increased significantly from $325.1 \mathrm{cc}$ at baseline to $391.2 \mathrm{cc}$ at 12 weeks and $371.2 \mathrm{cc}$ (not significant) at 24 weeks. More important from the patient standpoint were the decrease in frequency, nocturia, and the overall control of their bladder symptoms. Frequency decreased from 9 to 8.1 and 7.4 times per day at 12 and 24 weeks respectively and nocturia decreased from 1.9 to 1.0 and 1.5 times per night at 12 and 24 weeks. Furthermore, 5/7 patients, through questionnaires, reported improved urinary control while $6 / 7$ stated that they would undergo this treatment again. While this paper does not include patients with detrusor overactivity, it suggests that the BTX injections may have even more applications in refractory urinary symptoms than originally suggested.

The most recent study, reported in July of 2006 by Schmid et al. [9] in an ongoing prospective, nonrandomized trial evaluating 100 patients with overactive bladder symptoms, with and without detrusor instability after injection of $100 \mathrm{U}$ BTX-A. At 4, 12, and 36 weeks post-injection, these patients were evaluated for urodynamic and quality of life parameters to determine both the effectiveness of this treatment at this dose and effectiveness of the injections in 2 subtypes of patients with refractory symptoms (with and without detrusor instability). In terms of quality of life as evaluated by the $\mathrm{KHQ}, 90 \%$ of patients showed improvement in at least 1 category at both 4 and 12 weeks. This positive outcome was also significant at 36 weeks, but was slightly diminished given the reversibility of BTX-A. Subjectively, $88 \%$ reported improvement in bladder function, $80 \%$ of those who were incontinent were dry and urgency disappeared in $66 \%$ of patients at 12 weeks. Additionally a significant decrease in frequency and nocturia was noted throughout patient types. In terms of urodynamics, a significant correlation was found between clinically observed symptoms and changes in urodynamic parameters. The mean MCBC increased from 246 cc to 381 cc (56\% increase) at 12 weeks, the mean volume at first urge to void increased from $126 \mathrm{cc}$ to $212 \mathrm{cc}$ and the MBC increased from 24 to $41 \mathrm{cc} / \mathrm{cmH}_{2} \mathrm{O}$. While these values remained elevated a decline was noted at 36 weeks. Both daytime frequency and nocturia were significantly reduced and persisted for the three months (Table 3). No prolonged side effects were noted; however 4 patients did develop transient urinary retention with elevated PVRs, requiring temporary ISC. This issue was resolved with time and had little effect on symptomatic 
improvement. By and large, this study is well done and sheds a bit of light on some of the more pressing questions in the study of BTX in IDO. Overall it suggests that $100 \mathrm{U}$ dose injections are quite effective in measures of both objective and subjective success and confirms that BTX-A is useful in both patients with and without idiopathic detrusor instability.

\section{Head to head trial: BTX-A effectiveness in neurogenic vs. idiopathic bladder dysfunction}

Although individually, BTX-A has been shown to be effective in both neurogenic and idiopathic detrusor overactivity, there are very few studies directly comparing the efficacy in a mixed population. Popat et al. in 2005 [13] studied 44 patients with NDO and 31 patients with IDO that had been injected with $300 \mathrm{U}$ and $200 \mathrm{U}$ of BTX-A respectively and evaluated for urodynamics and subjective outcomes for a total of 16 weeks. All patients reported improvement in urinary control and approximately $63 \%$ of patients reported continence at 16 weeks. Although it initially it seemed as if continence rates were better in the NDO group at 4 weeks, no difference was shown between the groups at 16 weeks, suggesting that BTX-A is equally clinically effective in both patient populations. Urodynamic evaluations were also much improved in both groups at 16 weeks. The mean MCBC in the IDO group increased from $193.6 \mathrm{cC}$ to $327.1 \mathrm{cc}$ and increased from $229.1 \mathrm{cc}$ to $427.0 \mathrm{cc}$ in the NDO group, results that are significantly different from baseline, but not significantly different between groups. Results were similar for mean bladder pressure, frequency and urge episodes. Taken as a whole, this study both confirms previous investigations implicating BTX-A as a treatment for detrusor overactivity as well as reporting equivalent efficacy in both patient populations.

\section{Botulinum toxin-B}

In addition to the examination of Botulinum toxintype $A$ in the management of detrusor overactivity, Botulinum toxin-type $B$ has also been investigated. This subtype has a similar mechanism of action as the A type, but cleaves a different target protein $[1,15]$ making it a potential therapeutic option for treatment of patients unresponsive to toxin-type A or for those patients who become resistant to repeated toxin-A injections. The first urologic case using BTX-B was reported by Dykstra et al. in 2003 [14]. In this study, 15 patients with detrusor hyperreflexia were
Table 3 Urodynamic data after $100 \mathrm{U}$ injections in patients with $\mathrm{OAB}$ : clinical parameters

\begin{tabular}{|c|c|c|c|c|}
\hline \multirow[t]{2}{*}{ Parameter } & \multirow{2}{*}{$\begin{array}{l}\text { Mean } \\
\text { no. } \\
\text { preop } \\
\pm \text { SD }\end{array}$} & \multicolumn{3}{|c|}{ Mean no. postop \pm SD } \\
\hline & & 1 month & 3 months & 9 months \\
\hline No. pts & 100 & 100 & 80 & 20 \\
\hline $\begin{array}{l}\text { Daytime } \\
\text { frequency }\end{array}$ & $13.5 \pm 0.6$ & $7 \pm 0.8^{*}$ & $7 \pm 0.8^{*}$ & $8 \pm 2.4$ \\
\hline Nocturia & $4 \pm 0.25$ & $1.6 \pm 0.3^{*}$ & $1.6 \pm 0.3^{*}$ & $2.1 \pm 0.9$ \\
\hline
\end{tabular}

treated with doses of BTX-B ranging from 2500 to $15,000 \mathrm{U}$. Outcomes were measured in terms of subjective improvement in symptoms and upon treatment, patients were found to have 5.27 fewer frequency episodes per day. The longest duration of the effect was 12 weeks, a result that highly correlated with dose. A more extensive study performed in 2005 examined the effect of $5000 \mathrm{U}$ BTX-B in 20 patients with neurogenic and idiopathic detrusor overactivity vs. placebo with treatment crossover after 6 weeks [15]. The primary outcome of this study was average voiding volume, while the secondary outcomes were frequency, incontinence episodes, and QOL as evaluated by the KHQ. Across all outcome measures, BTX-B was found to be significantly superior to placebo, supporting earlier data suggesting that BTX-B could be used for treatment of detrusor overactivity in addition to BTX-A.

Another question, however, is whether BTX-B can be used in cases refractory to or resistant to repeated BTX-A injections. Resistance to BTX-A has been reported and is postulated to be caused by an antibody response to the specific toxin subtype, resulting in a decreased response with subsequent injections, eventually rendering this treatment ineffective [15]. A case study performed by Reitz et al. [16] in 2004 suggests that BTX-B may be the answer to this problem. In this case report, 2 patients with neurogenic detrusor overactivity, who became resistant to BTX-A injections after multiple treatments were then treated with BTX-B. (Resistance was measured by the extensor digitorum brevis test in which motor potentials were measured before and after BTX-A injections.) In both cases urodynamic and clinical outcomes after $7500 \mathrm{U}$ of BTX-B showed benefits similar to those demonstrated with BTX-A which lasted a mean of 5.5 months with no observed side effects. Additional investigation is needed to increase the strength of observation but this study lends optimism for the use of BTX-B in BTX-A resistant patients. 


\section{Costs}

When considering new treatment options for any disease process, cost benefit analysis is an important component in determining the treatment's widespread applications. In the case of BTX injections, it has been proven to be safe and effective in the treatment of detrusor overactivity without having to proceed to more invasive procedures, but is the cost worth the results? It is estimated that the cost of one intra-office treatment session of BTX-A with intravesical anesthesia is $\sim \$ 1000$ every 6 months. If compared to surgical management, with the many thousands of dollars in costs accrued in the $\mathrm{OR}$ and with subsequent follow up and complications, BTX-A may be cost effective. However, since the FDA has not yet approved Botox for the treatment of detrusor overactivity, insurance companies seldom cover this cost, leaving many physicians desperate to find ways to fund this treatment to their patients. While this is unfortunate, it is only with further research that BTX for detrusor overactivity will become an FDA approved application.

\section{Conclusion}

Current management of both idiopathic and neurogenic detrusor overactivity is focused on either medical management with anticholinergic medications or more invasive measures such as bladder augmentation and nerve stimulation to decrease the debilitating symptoms of detrusor dysfunction. However, when medical management fails and surgery seems excessive, alternative treatment options are needed. In the search for alternative measures, Botulinum toxin-A has emerged as a promising option. Studies to date have shown that not only is this treatment effective at decreasing urinary symptoms and incontinence, as well as improving potentially dangerous urodynamic measures, but it is also minimally invasive, reversible and safe. Questions over proper dosing and dilution, injection sites, and re-injection rates remain to be answered. Additionally, its short duration of action (12-36 weeks), the proposed development of resistance, as well as the possible need for general anesthesia, makes its widespread use undefined at this time. Current and ongoing studies are now focusing on objective and subjective improvements as well as the posed unanswered questions regarding this treatment in a refractory patient population. Although there still is work to be done, the future of BTX-A use in management of detrusor overactivity is hopeful.

\section{References}

[1] Frenkl TL, Rackley RR. Injectable neuromodulatory agents: botulinum toxin therapy. Urol Clin North Am 2005;32:89-99.

[2] Bullock TL, Klutke CG, Klutke JJ. Botulinum toxin in the treatment of neurogenic and idiopathic urinary incontinence. Female Patient 2006;31:14-24.

[3] Reitz A, Stohrer M, Kramer G. European experience of 200 cases treated with botulinum-A toxin injections into the detrusor muscle for urinary incontinence due to neurogenic detrusor overactivity. Eur Urol 2004;45:510-5.

[4] The standardization of terminology in lower urinary tract function: report from the Standardization Sub-committee of the International Continence Society. Urology 2003;61:37-49.

[5] Schurch B, Stohrer M, Kramer G. Botulinum-A toxin for treating detrusor hyperreflexia in spinal cord injured patients: a new alternative to anticholinergic drugs? Preliminary results. J Urol 2000;164:692-7.

[6] Bagi P, Biering-Sorensen F. Botulinum toxin A for treatment of neurogenic detrusor overactivity and incontinence in patients with spinal cord lesions. Scand J Urol Nephrol 2004;38:495-8.

[7] Klaphajone J, Kitisomprayoonkul W, Sriplakit S. Botulinum toxin type A injections for treating neurogenic detrusor overactivity combined with low-compliance bladder in patients with spinal cord lesions. Arch Phys Med Rehabil 2005;86:2114-8.

[8] Schurch B, DeSeze M, Denys P. Botulinum toxin type A is a safe and effective treatment for neurogenic urinary incontinence: results of a singe treatment, randomized, placebo controlled 6 mo study. J Urol 2005;174:196-200.

[9] Schmid DM, Sauermann P, Werner M, Schuessler B, Blick N, Muentener $M$, et al. Experience with 100 cases treated with botulinum-A toxin injections in the detrusor muscle for idiopathic overactive bladder syndrome refractory to anticholinergics. J Urol 2006;176:177-85.

[10] Werner M, Schmid D, Schussler B. Efficacy of botulinum-A toxin in the treatment of detrusor overactivity incontinence: a prospective nonrandomized study. AJOG 2005; 192:1735-40.

[11] Kuo H. Clinical effects of suburothelial injection of botulinum A toxin on patients with nonneurogenic detrusor overactivity refractory to anticholinergics. Urology 2005; 66:94-8.

[12] Schulte-Baukloh $\mathrm{H}$, Weiss $\mathrm{C}$, Stolze T. Botulinum-A toxin for treatment of overactive bladder without detrusor overactivity: urodynamic outcome and patient satisfaction. Urology 2005;66:81-7.

[13] Popat R, Apostolidis A, Kalsi V. A comparison between the response of patients with idiopathic detrusor overactivity and neurogenic detrusor overactivity to the first intradetrusor injection of botulinum-A toxin. J Urol 2005;174:984-9.

[14] Dykstra D, Enriquez A, Valley M. Treatment of overactive bladder with botulinum toxin type B: a pilot study. Int Urogynecol J 2003;14(6):424-6.

[15] Ghei M, Maraj BH, Miller R. Effects of botulinum toxin B on refractory detrusor overactivity: a randomized, double-blind, placebo controlled, crossover trial. J Urol 2005;174:1873-7.

[16] Reitz A, Schurch B. Botulinum toxin type B injection for management of type $A$ resistant neurogenic detrusor overactivity. J Urol 2004;171:804-5. 